Article

\title{
The Brownian and Thermophoretic Analysis of the Non-Newtonian Williamson Fluid Flow of Thin Film in a Porous Space over an Unstable Stretching Surface
}

\author{
Liaqat Ali ${ }^{1,2}$, Saeed Islam ${ }^{1}$, Taza Gul ${ }^{1}$, Ilyas Khan ${ }^{3}$, L. C. C. Dennis ${ }^{4, *}$, Waris Khan ${ }^{5}$ and \\ Aurangzeb Khan 6 \\ 1 Department of Mathematics, Abdul Wali Khan University, Mardan 23200, KPK, Pakistan; \\ liaqat@cecos.edu.pk (L.A.); saeedislam@awkum.edu.pk (S.I.); tazagulsafi@yahoo.com (T.G.) \\ 2 Department of Electrical Engineering, CECOS University, Peshawer 25000, KPK, Pakistan \\ 3 Department of Mathematics, College of Engineering, Majmaah University, Majmaah 31750, Saudi Arabia; \\ ilyaskhanqau@yahoo.com \\ 4 Department of Fundamental and Applied Sciences, Universiti Teknologi Petronas, Perak 32610, Malaysia \\ 5 Department of Mathematics, Islamia College, Peshawer 25000, KPK, Pakistan; wariskhan758@yahoo.com \\ 6 Department of Physics, Abdul Wali Khan University, Mardan 23200, KPK, Pakistan; akhan@awkum.edu.pk \\ * Correspondence: dennis.ling@petronas.com.my; Tel.: +60-168-529-529-339
}

Academic Editor: Richard Yong Qing Fu

Received: 19 February 2017; Accepted: 5 April 2017; Published: 18 April 2017

\begin{abstract}
This paper explores Liquid Film Flow of Williamson Fluid over an Unstable Stretching Surface in a Porous Space. The Brownian motion and Thermophoresis effect of the liquid film flow on a stretching sheet have been observed. This research include, to focus on the variation in the thickness of the liquid film in a porous space. The self-similarity variables have been applied to convert the modelled equations into a set of non-linear coupled differential equations. These non-linear differential equations have been treated through an analytical technique known as Homotopy Analysis Method (HAM). The effect of physical non-dimensional parameters like, Eckert Number, Prandtl Number, Porosity Parameter, Brownian Motion Parameter, Unsteadiness Parameter, Schmidt Number, Thermophoresis Parameter, Dimensionless Film Thickness, and Williamson Fluid Constant on the liquid film size are investigated and conferred in this endeavor. The obtained results through HAM are authenticated, from its comparison with numerical (ND-Solve Method). The graphical comparison of these two methods is elaborated. The numerical comparison with absolute errors are also been shown in the tables. The physical and numerical results using $h$ curves for the residuals of the velocity, temperature and concentration profiles are obtained.
\end{abstract}

Keywords: Thermophoretic effect and Brownian motion, thin film, porous medium, Williamson fluid, unsteady stretching sheet, HAM, ND-solve methods

\section{Introduction}

In the existing literature most of the study is related to Newtonian Fluids and very little attention is paid to the Non-newtonian fluids. Therefore Williamson Fluid has been selected from the class of non-newtonian shear thickening and shear thinning fluids, which has many uses in the field of industry and engineering. The flow of Pseudoplastic Fluids experimentally describe by Williamson [1] with verified results. The analytical study of Williamson Fluid can be found in the investigation of Dapra and Scarpi [2]. Thermophoresis (also Thermomigration, Thermodiffusion, the Soret Effect, or the Ludwig-Soret Effect) is a phenomenon observed in mixtures of mobile particles where the different particle types exhibit different responses, to the force of a temperature gradient. The term Thermophoresis most often applies to aerosol mixtures, but may also commonly refer to the 
phenomenon in all forms of matter. The term Soret Effect normally applies to liquid mixtures, which behave in different, less well-understood mechanisms than gaseous mixtures. Thermophoresis may not apply to thermomigration in solids, especially multi-phase alloys. The phenomenon is observed at the scale of one millimeter or less. An example that may be observed by the naked eye with good lighting is when the hot rod of an electric heater is surrounded by tobacco smoke, the smoke goes away from the immediate vicinity of the hot rod. As the small particles of air nearest the hot rod are heated, they create a fast flow away from the rod, down the temperature gradient. They have acquired higher kinetic energy with their higher temperature. When they collide with the large, slower-moving particles of the tobacco smoke they push the latter away from the rod. The force that has pushed the smoke particles away from the rod is an example of a Thermophoretic Force. Brownian motion or Pedesis is the random motion of particles suspended in a fluid (a liquid or a gas) resulting from their collision with the fast-moving atoms or molecules in the gas or liquid. Transfer of heat energy play an important role in almost all of the industrial processes. It is used to save energy and reduce processing time in industrial processes. It is also used to raise the thermal rating and increase the working life of equipment. The Liquid Film Flow of Williamson Fluid in a Porous Space over an Unstable Stretching Surface has focused the interest of several researchers because of its many uses in the fields of engineering and industries. The hydrodynamics of a thin liquid film over an unsteady stretching sheet is studied by Wang et al. [3] and Cramer et al. [4] for the first time. The effect of surface mass transfer mixed convection flow is explored by Selim et al. [5]. Das [6] analyzed the impact of thermal radiation on MHD slip flow over a flat plate with variable fluid properties. The effects of radiation and heat transfer on MHD flow of Viscoelastic Liquid and heat transfer over a stretching sheet is studied by Siddeshwar et al. [7]. Nadeem and Hussain [8] solved the problem of flow and heat transfer analysis of Williamson Nanofluid. Hassanien et al. [9] worked on Variable viscosity and thermal conductivity effects on heat transfer by natural convection from a cone and a wedge in porous media. Aziz et al. [10] considered thin film flow and heat transfer on an unsteady stretching sheet with internal heating. Qasim et al. [11] used Buongiorno's model to investigate heat and mass transfer in Nanofluid. Mahesh et al. [12] studied Heat and Mass Transfer in Nanofuid over an unsteady stretching surface.

Ellahi et al. studied Nanofluid over different phenomena mentioned in [13-17]. A detailed data on thin film Williamson Nanofluid Flow with Varying Viscosity and Thermal Conductivity on a Time-Dependent Stretching Sheet is given by Khan et al. [18]. The present research is the study of liquid film flow of Williamson Fluid in a porous medium over an unsteady stretching sheet with the combined effect of Thermophoresis and Brownian motion. The self-similarity variables has been used to convert the modelled equations into a set of non-linear coupled differential equations. The flow of fluid in a porous medium has also a significant role in the field of engineering and especially in Bio-engineering. The purification of liquids through filtration, human lungs, blood filtration are the application of porous media. The flow of fluid in a porous medium on a stretching sheet can be seen in $[19,20]$. These non-linear differential equations has been tackled through a powerful analytical method known as Homotopy Analysis Method (HAM) [21-28]. The relevant work can also be found in [29-35]. The effect of physical non-dimensional parameters like Porosity Parameter, Unsteadiness Parameter, Prandtl Number, Schmidt Number, and Dimensionless Film thickness on the liquid film size has been investigated and discussed. The results achieved by the HAM and numerical ND-Solve method are compared and presented in the form of figures and tables with absolute error to make understandable for readers.

\section{Mathematical Formulation of Model}

Suppose a two dimensional incompressible Liaquid Film Flow of Williamson Fluid on a Porous Unsteady Stretching Sheet with thermal radiation, where heat and mass are transferred simultaneously. The coordinate axes are chosen in such away that the $x$-axis is parallel to the plate while the $y$-axis is perpendicular to it. The stretching velocity of the sheet is in the direction of the $x$-axis which have magnitude $U_{w}=\frac{\alpha x}{1-\gamma t}$, in which $\alpha>0$ is the stretching velocity constraint and $\gamma \in[0,1]$. 
The temperature $T_{w}(x, t)=T_{0}-T_{r e f}\left(\frac{\alpha x^{2}}{2 v}\right)(1-\gamma t)^{-1.5}$, where $T_{0}$ elaborates the temperature at the surface and $T_{r e f}$ depicts the reference temperature. Similarly, $C_{w}(x, t)=C_{0}-C_{r e f}\left(\frac{\alpha x^{2}}{2 v}\right)(1-\gamma t)^{-1.5}$ is the volume concentration, where $C_{0}$ illustrates the concentration at the surface and $C_{r e f}$ shows the reference concentration. The time dependent term $\frac{\alpha x^{2}}{v(1-\gamma t)}$, indicates the local Reynold number which reliant on the stretching velocity $U_{w}(x, t)$. Initially the sheet is fixed with the origin and then an external force is applied to stretch the surface in the positive $x$-axis at the rate $\frac{\alpha}{(1-\gamma t)}$ in time $t$ with velocity $U_{w}(x, t)$, where $\gamma \in[0,1]$. Now use the above conditions, to get the following equations as:

Continuity Equation,

$$
\frac{\partial u}{\partial x}+\frac{\partial v}{\partial y}=0
$$

Momentum Equation,

$$
\frac{\partial u}{\partial t}+u \frac{\partial u}{\partial x}+v \frac{\partial u}{\partial y}=v \frac{\partial^{2} u}{\partial y^{2}}+2^{0.5} \Gamma v \frac{\partial^{2} u}{\partial y^{2}} \frac{\partial u}{\partial y}-\frac{v \phi}{K} u,
$$

Energy Equation,

$$
\frac{\partial T}{\partial t}+u \frac{\partial T}{\partial x}+v \frac{\partial T}{\partial y}=\alpha \frac{\partial^{2} T}{\partial y^{2}}+\tau\left[D_{B}\left(\frac{\partial C}{\partial y} \frac{\partial T}{\partial y}\right)+\frac{D_{T}}{T_{\infty}}\left(\frac{\partial T}{\partial y}\right)^{2}\right]+\frac{v}{C_{p}}\left[\left(\frac{\partial u}{\partial y}\right)^{2}+2^{0.5} \Gamma\left(\frac{\partial u}{\partial y}\right)^{3}\right],
$$

Concentration Equation,

$$
\frac{\partial C}{\partial t}+u \frac{\partial C}{\partial x}+v \frac{\partial C}{\partial y}=D_{B} \frac{\partial^{2} C}{\partial y^{2}}+\frac{D_{T}}{T_{\infty}}\left(\frac{\partial^{2} T}{\partial y^{2}}\right)
$$

along with the BCs,

$$
\begin{aligned}
& u=U_{w}, T=T_{w}, v=0, C=C_{w}, y=0, \\
& \frac{\partial u}{\partial y}=\frac{\partial C}{\partial y}=\frac{\partial T}{\partial y}=0, v=\frac{d h}{d t}=0, y=h(t) .
\end{aligned}
$$

$u$ and $v$ are the flow velocities along $x$ and $y$ axis respectively, the Specific heat at constant pressure is represented by $C_{p}$, the Thermal diffusivity of the base fluid is indicated by $\alpha=\frac{k}{(\rho c) p}, \Gamma>0$ is the Time constant, the Fluid density is represented by $\rho c, \tau=\frac{(\rho c) p}{(\rho c) f}$ and the local nanoparticle Volume fraction is denoted by $C$. Also the Thermophoretic diffusion coefficient is indicated by $D_{T}, \rho$ is the Density, while the Brownian diffusion coefficient is shown by $D_{B} . T$ is the local Temperature and the Film thickness is denoted by $h(t)$.

Now define the following similarity transformations as:

$$
\begin{aligned}
& \xi=\left(\frac{\alpha}{v(1-\gamma t)}\right)^{0.5} y, \\
& \psi(x, y, t)=\left(\frac{v \alpha}{1-\gamma t}\right)^{0.5} x f(\xi), \\
& T(x, y, t)=T_{0}-T_{r e f}\left(\frac{\alpha x^{2}}{2 v}\right)(1-\gamma t)^{-1.5} \theta(\xi), \\
& C(x, y, t)=C_{0}-C_{r e f}\left(\frac{\alpha x^{2}}{2 v}\right)(1-\gamma t)^{-1.5} \phi(\xi) .
\end{aligned}
$$

$\psi(x, y)$ is the Stream function which is defined as: $u=\frac{\partial \psi}{\partial y}, v=-\frac{\partial \psi}{\partial x} \cdot \beta$ is Non-dimensional film thickness and is described as $\beta=\left(\frac{\alpha}{v(1-\gamma t)}\right)^{0.5}(h(t))[29,30]$. 
Also $\frac{d h}{d t}=-\frac{\gamma}{2} \beta\left(\frac{v}{\alpha}\right)^{0.5}(1-\gamma t)^{-0.5}$. Now put the values in the above equations we get a system of nonlinear coupled boundary value problems as:

$$
\begin{gathered}
\frac{d^{3} f(\xi)}{d \xi^{3}}+\lambda \frac{d^{2} f(\xi)}{d \xi^{2}} \frac{d^{3} f(\xi)}{d \xi^{3}}+f(\xi) \frac{d^{2} f(\xi)}{d \xi^{2}}-\left(\frac{d f(\xi)}{d \xi}\right)^{2}-S\left(\frac{d f(\xi)}{d \xi}+\frac{\xi}{2} \frac{d^{2} f(\xi)}{d \xi^{2}}\right)-K_{r} \frac{d f(\xi)}{d \xi}=0, \\
\frac{d^{2} \theta(\xi)}{d \xi^{2}}+\operatorname{Pr} f(\xi) \frac{d \theta(\xi)}{d \xi}-2 \operatorname{Pr} \frac{d f(\xi)}{d \xi} \theta(\xi)-\operatorname{Pr}\left(\frac{\mathcal{S}}{2}\left(3 \theta(\xi)+\xi \frac{d \theta(\xi)}{d \xi}\right)\right)+ \\
\left.\operatorname{Pr} N b \frac{d \phi(\xi)}{d \xi} \frac{d \theta(\xi)}{d \xi}\right)+\operatorname{Pr} N t\left(\frac{d \theta(\xi)}{d \xi}\right)^{2}+\operatorname{Pr} E_{\mathcal{c}}\left(\left(\frac{d^{2} f(\xi)}{d \xi^{2}}\right)^{2}+\lambda\left(\frac{d^{2} f(\xi)}{d \xi^{2}}\right)^{3}\right)=0, \\
\frac{d^{2} \phi(\xi)}{d \xi^{2}}+S c\left(\frac{d \phi(\xi)}{d \xi} f(\xi)-2 \frac{d f(\xi)}{d \xi} \phi(\xi)-\frac{S}{2}\left(3 \phi(\xi)+\xi \frac{d \phi(\xi)}{d \xi}\right)+\frac{N t}{N b} \frac{d^{2} \theta(\xi)}{d \xi^{2}}=0,\right.
\end{gathered}
$$

along with transformed boundary conditions,

$$
\frac{d^{2} f(\beta)}{d \xi^{2}}=0, \frac{d f(0)}{d \xi}=1, f(0)=0, f(\beta)=\frac{S \beta}{2}, \frac{d \theta(\beta)}{d \xi}=0, \theta(0)=1, \frac{d \phi(\beta)}{d \xi}=0, \phi(0)=1 .
$$

where

$$
\begin{gathered}
\lambda=\Gamma U_{w}\left(\frac{2 \alpha}{v(1-\gamma t)}\right)^{0.5}, K_{r}=\frac{v^{2} \phi(1-\gamma t)}{\alpha K}, \\
S=\frac{\gamma}{\alpha}, \operatorname{Pr}=\frac{v \rho C_{p}}{k}, E_{c}=\frac{U_{w}^{2}}{C_{p}\left(T_{w}-T_{0}\right)}, \\
S c=\frac{v}{D_{B}}, N b=\tau D_{B}\left(C_{w}-C_{\infty}\right), N t=\frac{\tau D_{T}\left(T_{w}-T_{\infty}\right)}{v T_{\infty}} .
\end{gathered}
$$

\section{Materials and Methods}

In this section high accuracy of the applied method is applied to system of nonlinear boundary value problems obtained from the new modeled phenomenon. As a result, we see that this method gives best approximation and takes very less time to produce good results.

\section{Solution of Problem}

For the solution of system (7) an analytical technique, called Homotopy Analysis Method (HAM) is used. To apply this method we first find the initial guesses $f_{0}(\xi), \theta_{0}(\xi), \phi_{0}(\xi)$ from the following as: Zeroth order problem:

$$
\begin{gathered}
\frac{d^{3} f_{0}(\xi)}{d \xi^{3}}=0, f_{0}(0)=0, \frac{d f_{0}(0)}{d \xi}=1, \frac{d^{2} f_{0}(\beta)}{d \xi^{2}}=0, \\
\frac{d^{2} \theta_{0}(\xi)}{d \xi^{2}}=0, \theta_{0}(0)=1, \frac{d \theta_{0}(\beta)}{d \eta}=0, \\
\frac{d^{2} \phi_{0}(\xi)}{d \xi^{2}}=0, \phi_{0}(0)=1, \frac{d \phi_{0}(\beta)}{d \xi}=0,
\end{gathered}
$$

which gives the solution as

$$
f_{0}(\xi)=\xi, \theta_{0}(\xi)=1, \phi_{0}(\xi)=1
$$

The linear operators are chosen as $\psi_{f}=\frac{d^{3} f(\mathcal{\zeta})}{d \xi^{3}}, \quad \psi_{\theta}=\frac{d^{2} \theta(\mathcal{\xi})}{d \xi^{2}}$ and $\psi_{\phi}=\frac{d^{2} \phi(\xi)}{d \xi^{2}}$ with the following properties

$$
\psi_{f}\left(C_{1}+C_{2} \xi+C_{3} \xi^{2}\right)=0, \psi_{\theta}\left(C_{4}+C_{5} \xi\right)=0, \psi_{\phi}\left(C_{6}+C_{7} \xi\right)=0,
$$

where $C_{i}, i=1-7$ are constants. The resultant non-linear operators $\aleph_{f}, \aleph_{\theta}$ and $\aleph_{\phi}$ are chosen as:

$$
\aleph_{f}(\xi ; \wp)=\frac{d^{3} f(\xi)}{d \xi^{3}}+f(\xi) \frac{d^{2} f(\xi)}{d \xi^{2}}+\lambda \frac{d^{2} f(\xi)}{d \xi^{2}} \frac{d^{3} f(\xi)}{d \xi^{3}}-\left(\frac{d f(\xi)}{d \xi}\right)^{2}-S\left(\frac{d f(\xi)}{d \xi}+\frac{\xi}{2} \frac{d^{2} f(\xi)}{d \xi^{2}}\right)-K_{r} \frac{d f(\xi)}{d \xi},
$$




$$
\begin{gathered}
\aleph_{\theta}[f(\xi ; \wp), \theta(\xi ; \wp), \phi(\xi ; \wp)]=\frac{d^{2} \theta(\xi)}{d \xi^{2}}+\operatorname{Pr} f(\xi) \frac{d \theta(\xi)}{d \xi}-2 \operatorname{Pr} \frac{d f(\xi)}{d \xi} \theta(\xi)-\operatorname{Pr}\left(\frac{\mathcal{S}}{2}\left(3 \theta(\xi)+\xi \frac{d \theta(\xi)}{d \xi}\right)\right)+ \\
\left.\operatorname{Pr} N b \frac{d \phi(\xi)}{d \xi} \frac{d \theta(\xi)}{d \xi}\right)+\operatorname{Pr} N t\left(\frac{d \theta(\xi)}{d \xi}\right)^{2}+E_{\mathcal{c}}\left(\left(\frac{d^{2} f(\xi)}{d \xi^{2}}\right)^{2}+\lambda\left(\frac{d^{2} f(\xi)}{d \xi^{2}}\right)^{3}\right), \\
\aleph_{\phi}[f(\xi ; \wp), \theta(\xi ; \wp), \phi(\xi ; \wp)]=\frac{d^{2} \phi(\xi)}{d \xi^{2}}+S c\left(\frac{d \phi(\xi)}{d \xi} f(\eta)-2 \frac{d f(\xi)}{d \xi} \phi(\xi)-\right. \\
\left.\frac{S}{2}\left(3 \phi(\xi)+\xi \frac{d(\xi)}{d \xi}\right)\right)+\frac{N t}{N b} \frac{d^{2} \theta(\xi)}{d \xi^{2}} .
\end{gathered}
$$

The basic idea of HAM is described in [21-28],

Zeroth-order problems:

$$
\begin{gathered}
(1-\wp) \psi_{f}\left[f(\xi ; \wp)-f_{0}(\xi)\right]=\wp \hbar_{f} \aleph_{f}[f(\xi ; \wp)], \\
(1-\wp) \psi_{\theta}\left[\theta(\xi ; \wp)-\theta_{0}(\xi)\right]=\wp \hbar \aleph_{\theta}[f(\xi ; \wp), \theta(\xi ; \wp), \theta(\xi ; \wp)], \\
(1-\wp) \psi_{\phi}\left[\phi(\xi ; \wp)-\phi_{0}(\xi)\right]=\wp \hbar_{\phi} \aleph_{\phi}[f(\xi ; \wp), \theta(\xi ; p), \phi(\xi ; \wp)] .
\end{gathered}
$$

The equivalent $\mathrm{BCs}$ are:

$$
\begin{gathered}
f(0 ; \wp)=0, \frac{d f(0 ; \wp)}{d \zeta}=1, \frac{d^{2} f(\xi ; \wp)}{d \xi^{2}}=0, \\
\theta(0 ; \wp)=1, \frac{d \theta(0 ; \wp)}{d \zeta}=0, \phi(0 ; \wp)=1, \frac{d \phi(\beta ; \wp)}{d \zeta}=0 .
\end{gathered}
$$

where $\wp \in[0,1]$ is the imbedding parameter, $\hbar_{f}, \hbar_{\theta}$ and $\hbar_{\phi}$ are used to control the convergence of the solution. When $\wp=0$ and $\wp=1$, then:

$$
f(\xi ; 1)=f(\xi), \theta(\xi ; 1)=\theta(\xi), \phi(\xi ; 1)=\phi(\xi) .
$$

Expanding $f(\xi ; \wp), \theta(\xi ; \wp)$ and $\phi(\xi ; \wp)$ in Taylor's series about $\wp=0$ as:

$$
\begin{aligned}
& f(\xi)=f_{0}(\xi)+\sum_{m=0}^{m=\infty} f_{m}(\xi) \wp^{m}, \\
& \theta(\xi)=\theta_{0}(\xi)+\sum_{m=0}^{m=\infty} \theta_{m}(\xi) \wp^{m}, \\
& \phi(\xi)=\phi_{0}(\xi)+\sum_{m=0}^{m=\infty} \phi_{m}(\xi) \wp^{m} .
\end{aligned}
$$

where

$$
\begin{aligned}
& f_{m}(\xi)=\left.\frac{1}{m !} \frac{d^{m} f(\xi ; \wp)}{d \xi^{m}}\right|_{\wp=0} \\
& \theta_{m}(\xi)=\left.\frac{1}{m !} \frac{d^{m} \theta(\xi ; \zeta)}{d \xi^{m}}\right|_{\wp=0} \\
& \phi_{m}(\xi)=\left.\frac{1}{m !} \frac{d^{m} \phi(\xi ; \wp)}{d \xi^{m}}\right|_{\wp=0}
\end{aligned}
$$

The secondary constraints $\hbar_{f}, \hbar_{\theta}$ and $\hbar_{\phi}$ are selected in such away that the series (21) converges at $\wp=1$. Use $\wp=1$ in (21) to get:

$$
\begin{aligned}
& f(\xi)=f_{0}(\xi)+\sum_{m=0}^{m=\infty} f_{m}(\xi) \\
& \theta(\xi)=\theta_{0}(\xi)+\sum_{m=0}^{m=\infty} \theta_{m}(\xi) \\
& \phi(\xi)=\phi_{0}(\xi)+\sum_{m=0}^{m=\infty} \phi_{m}(\xi) .
\end{aligned}
$$


The $m^{\text {th }}$-order problem satisfies the following:

$$
\begin{aligned}
& \psi_{f}\left[f_{m}(\xi)-\chi_{m} f_{m-1}(\xi)\right]=\hbar_{f} R_{m}^{f}(\xi), \\
& \psi_{\theta}\left[\theta_{m}(\xi)-\chi_{m} \theta_{m-1}(\xi)\right]=\hbar_{\theta} R_{m}^{\theta}(\xi), \\
& \psi_{\phi}\left[\varphi_{m}(\xi)-\chi_{m} \phi_{m-1}(\xi)\right]=\hbar_{\phi} R_{m}^{\phi}(\xi) .
\end{aligned}
$$

The boundary conditions for this problem are:

$$
\begin{aligned}
& \frac{d^{2} f_{m}(\beta)}{d \xi^{2}}=0, \frac{d f_{m}(0)}{d \xi^{\tau}}=1, f_{m}(0)=0, f_{m}(\beta)=\frac{s \beta}{2}, \frac{d \theta_{m}(\beta)}{d \xi^{2}}=0, \theta_{m}(0)=1, \\
& \frac{d \phi_{m}(\beta)}{d \tilde{\zeta}}=0, \phi_{m}(0)=1 \text {. }
\end{aligned}
$$

Here

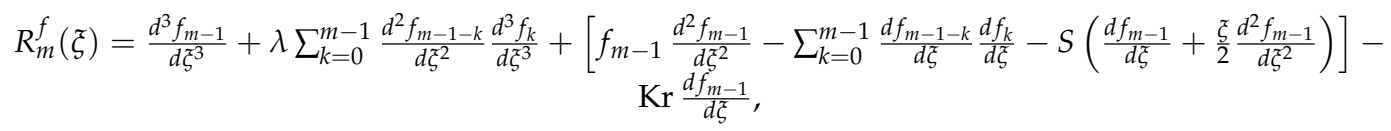

$$
\begin{aligned}
& R_{m}^{\theta}(\xi)=\frac{d^{2} \theta_{m-1}}{d \xi^{2}}+\operatorname{Pr}\left[-\frac{S}{2}\left(3 \theta_{m-1}+\xi \frac{d \theta_{m-1}}{d \xi}\right)-2 \sum_{k=0}^{m-1} \theta_{m-1-k} \frac{d f_{k}}{d \xi}+\sum_{k=0}^{m-1} f_{m-1-k} \frac{d \theta_{k}}{d \xi}\right]+ \\
& E_{C}\left[\sum_{k=0}^{m-1} \frac{d^{2} f_{m-1-k}}{d \xi^{2}} \frac{d^{2} f_{k}}{d \xi^{2}}+\lambda \sum_{k=0}^{m-1} \frac{d^{2} f_{m-1-k}}{d \xi^{2}} \sum_{\ell=0}^{k} \frac{d^{2} f_{k-1}}{d \xi^{2}} \frac{d^{2} f_{\ell}}{d \xi^{2}}\right]+N t \sum_{k=0}^{m-1} \frac{d \theta_{m-1-k}}{d \xi} \frac{d \theta_{k}}{d \xi^{2}} \\
& +N b\left(\frac{d \theta_{m-1}}{d \xi} \frac{d \phi_{m-1}}{d \xi}\right), \\
& R_{m}^{\phi}(\xi)=\frac{d^{2} \phi_{m-1}}{d \xi^{2}}+S c\left[\sum_{k=0}^{m-1} f_{m-1-k} \frac{d \phi_{j}}{d \xi}-2 \sum_{k=0}^{m-1} \frac{d f_{m-1-k}}{d \xi} \phi_{k}-\frac{S}{2}\left(3 \phi_{m-1}+\xi \frac{d \phi_{m-1}}{d \xi}\right)\right] \\
& +\frac{N t}{N b} \frac{d^{2} \theta_{m-1}}{d \xi^{2}}
\end{aligned}
$$

where

$$
\chi_{m}=\begin{aligned}
& 0, \text { if } \wp \leq 1 \\
& 1, \text { if } \wp>1
\end{aligned}
$$

\section{Representation of Achieved Results in the Form of Figures and Tables}

In this section the results achieved by HAM are shown in the form of figures and tables. The convergence of the series given in (21), $f(\eta), \theta(\eta)$ and $\phi(\eta)$ entirely depend upon the auxiliary parameters $\hbar_{f}, \hbar_{\theta}$ and $\hbar_{\phi}$ which are called $\hbar$-curves. It is selected in such a way that it controls and converges the series solution. The probable selection of $\hbar$ can be found by plotting $\hbar$-curves of $f^{\prime \prime}(0), \theta^{\prime}(0), \phi^{\prime}(0)$. The valid region of $\hbar$ is $-1.5<\hbar_{f}<-0.5,-1.5<\hbar_{\theta}<-0.5$ and $-1.5<\hbar_{\phi}<-0.5$. Here $\eta=\xi$ is chosen.

\section{Results and Discussion}

In this paper the Liquid Film Flow of Non-newtonian Williamson Fluid over an Unstable Stretching Surface in a Porous Space has been investigated. Thermophoresis and Brownian Motion Effect has been countered to the liquid film flow. The governing equations have been transformed through suitable similarity variables into nonlinear coupled differential equations with physical conditions. The solution of the coupled problem has been obtained by using an analytical approach called, HAM. The solution of the coupled problem and fast convergence of this method is mainly focused. This paper has examined the consequences of governing parameters on the transient velocity, temperature, and concentration profiles. Figure 1 illustrates the geometry of model used. Comparisons 
are carried out between the obtained results and the results achieved by numerical N-Desolve method for velocity, temperature, and concentration profiles (shown in Figures 2-4). The effects of physical parameters appear in the problem, are shown graphically and discussed. Figures 5-7, elaborate the behavior of the non-dimensional unsteady parameter $S$ for velocity, temperature and concentration field during fluid motion in a porous medium past over a Unsteady Stretching Sheet. The unsteady parameter $S$ is inversely related to the stretching constant of the velocity field, where as it is directly related to the stretching constants of the temperature and concentration fields. Therefore, when the values of $S$ are increasing the values of the velocity field are decreasing while the values of the temperature and concentration fields increase. Physically, unsteadiness $S$ produce buoyancy forces in the way of the flow field. These forces resist the fluid flow and therefore, the velocity field falls and the temperature distribution as well as the concentration profile is boosted. The effect of the Williamson Fluid constant $\lambda$ on the velocity field is illustrated in Figure 8 . The velocity is found to reduce when $\lambda$ is augmented. Because rise in relaxation time causes higher resistance to the fluid flow and as a result reduces the velocity field. Also increase in $\lambda$ increase the temperature due to increase in resistance to the fluid flow as shown in Figure 9. Non-dimensional porosity parameter $\mathrm{Kr}$ have direct relation to viscosity parameters. So a rise in non-dimensional porosity parameter reduces fluid motion as explained in Figure 10. Physically, larger values of $K r$ generate larger open space and create hurdle to flow and as a result the flow field is retarded. The resistance force produces larger values of $K r$ which increase the temperature and concentration profiles shown in Figures 11 and 12.

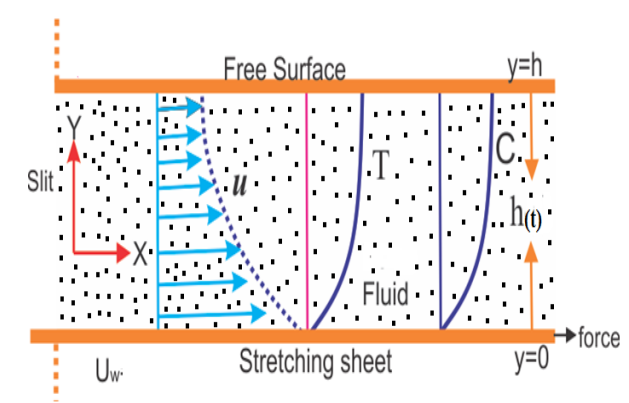

Figure 1. Illustrates the physical geometry of the used model.

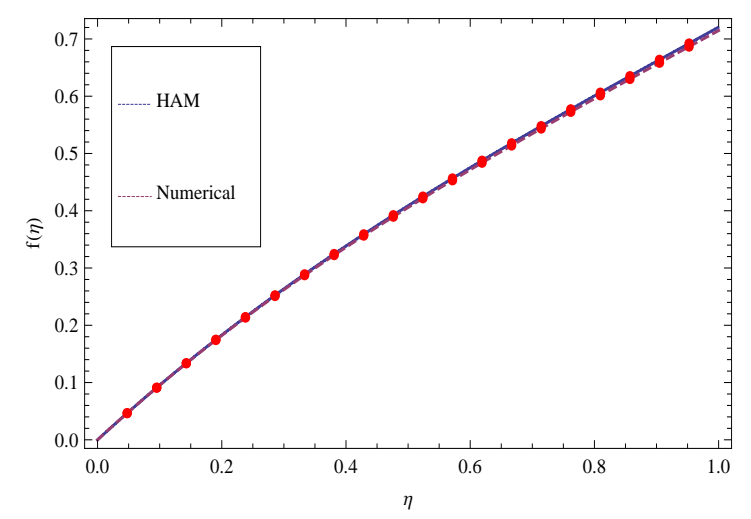

Figure 2. The comparison between HAM and numerical solutions for velocity profile $f(\eta)$, when $h=-0.25, \lambda=0.9, \mathrm{kr}=0.9, \operatorname{Pr}=0.5, \mathrm{Ec}=0.5, \mathrm{Nb}=0.5, \mathrm{Nt}=0.6, \beta=1$ and $S c=0.6$. 


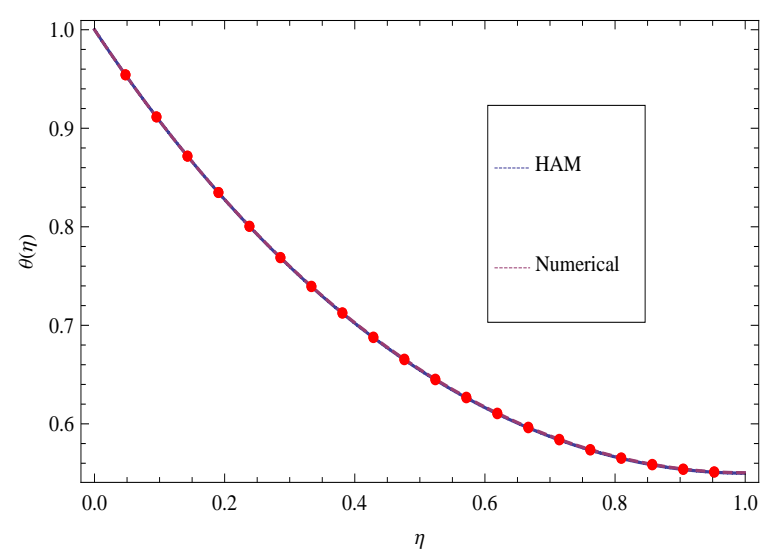

Figure 3. The comparison between HAM and numerical solutions for temperature profile $\theta(\eta)$, when $h=-0.47, \lambda=0.2, S=0.2, \mathrm{kr}=0.2, \operatorname{Pr}=1, \mathrm{Ec}=0.6, \mathrm{Nb}=0.4, \mathrm{Nt}=0.5, \beta=1, S c=0.5$.

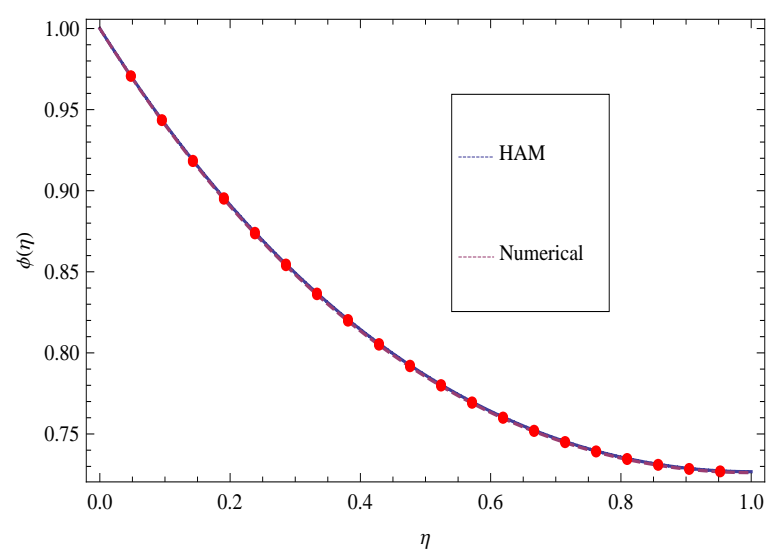

Figure 4. The comparison between HAM and numerical solutions for concentration profile $\phi(\eta)$, when $h=-0.6, \lambda=0.2, S=0.2, \mathrm{kr}=0.2, \mathrm{Pr}=1, \mathrm{Ec}=0.6, \mathrm{Nb}=1, \mathrm{Nt}=0.1, \beta=1$ and $S c=0.5$.

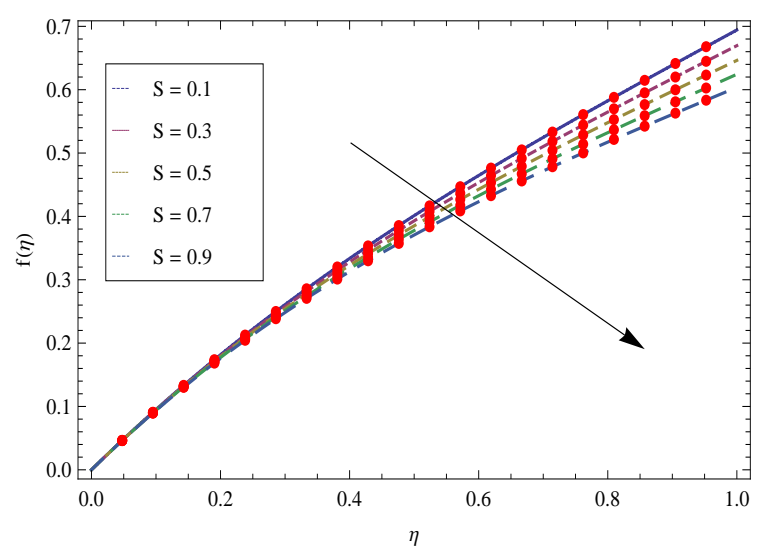

Figure 5. Variations in the Velocity field $f(\eta)$ for various values of $S$, when $h=-0.25, \lambda=0.9, \mathrm{kr}=0.9$, $\mathrm{Pr}=0.5, \mathrm{Ec}=0.5, \mathrm{Nb}=0.5, \mathrm{Nt}=0.6, \beta=1, \mathrm{Sc}=0.6$. 


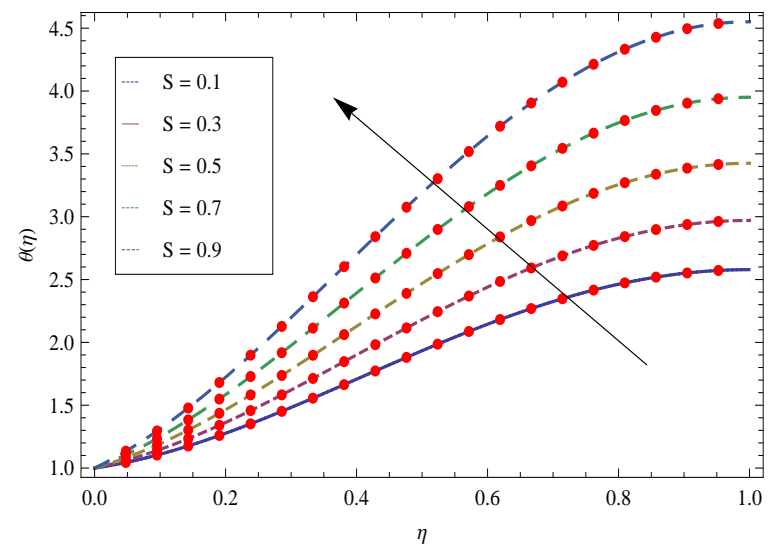

Figure 6. Variations in the Temperature gradient $\theta(\eta)$ for different values of $S$, when $h=-0.8, \lambda=0.1$, $\mathrm{kr}=0.5, \operatorname{Pr}=0.5, \mathrm{Ec}=0.5, \mathrm{Nb}=0.5, \mathrm{Nt}=0.6, \beta=1, \mathrm{Sc}=0.6$.

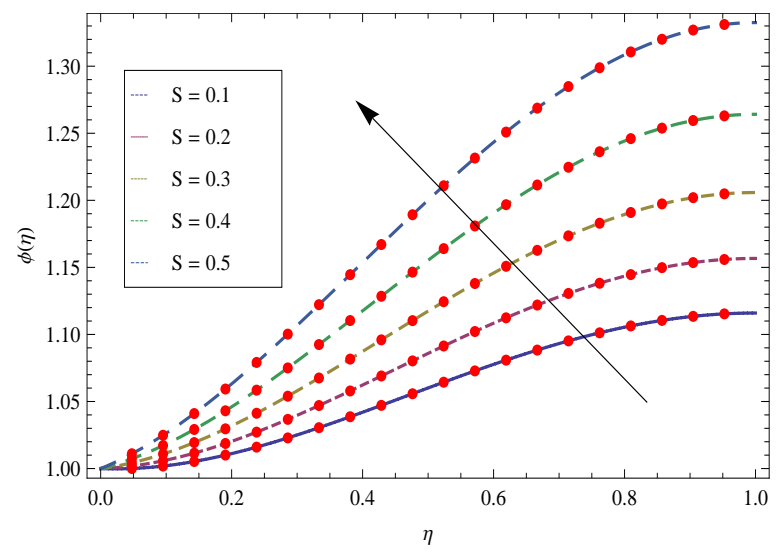

Figure 7. Variations in the Concentration field $\phi(\eta)$ for different values of $S$, when $h=-0.6, \lambda=0.5$, $\mathrm{kr}=0.5, \operatorname{Pr}=0.5, \mathrm{Ec}=0.5, \mathrm{Nb}=0.5, \mathrm{Nt}=0.6, \beta=1, \mathrm{Sc}=0.6$.

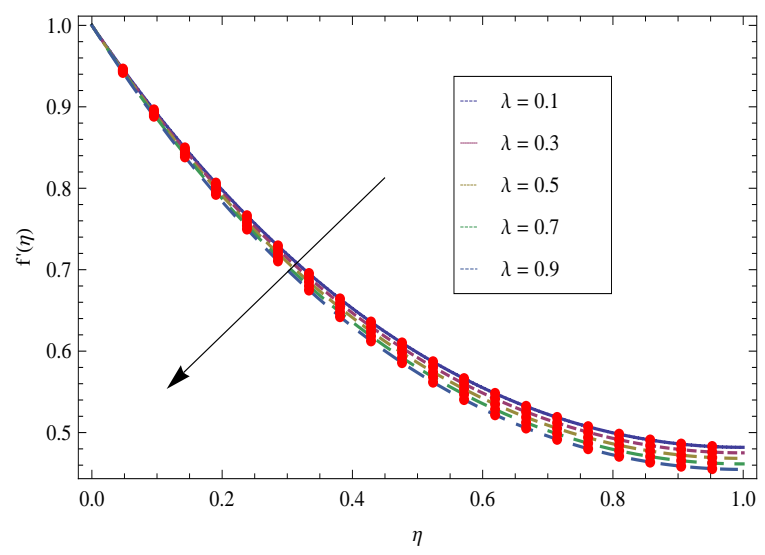

Figure 8. The effect of $\lambda$ on $f^{\prime}(\eta)$, when $h=-0.25, \mathrm{kr}=0.7, \mathrm{Pr}=0.5, \mathrm{Ec}=0.5, \mathrm{Nb}=0.5, \mathrm{Nt}=0.6$, $\beta=1, S c=0.6$. 


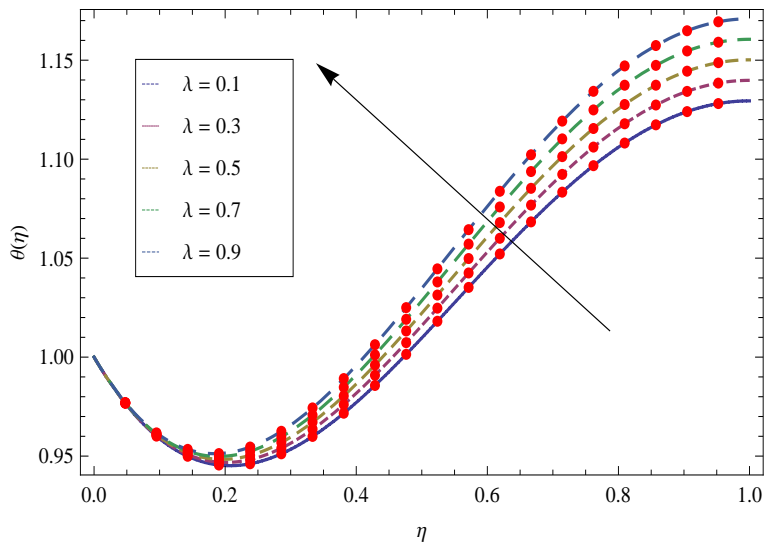

Figure 9. The effect of $\lambda$ on $\theta(\eta)$, when $h=-0.6, S=0.5, \mathrm{kr}=0.5, \operatorname{Pr}=0.5, \mathrm{Ec}=0.5, \mathrm{Nb}=0.5$, $\mathrm{Nt}=0.6, \beta=1, S c=0.6$.

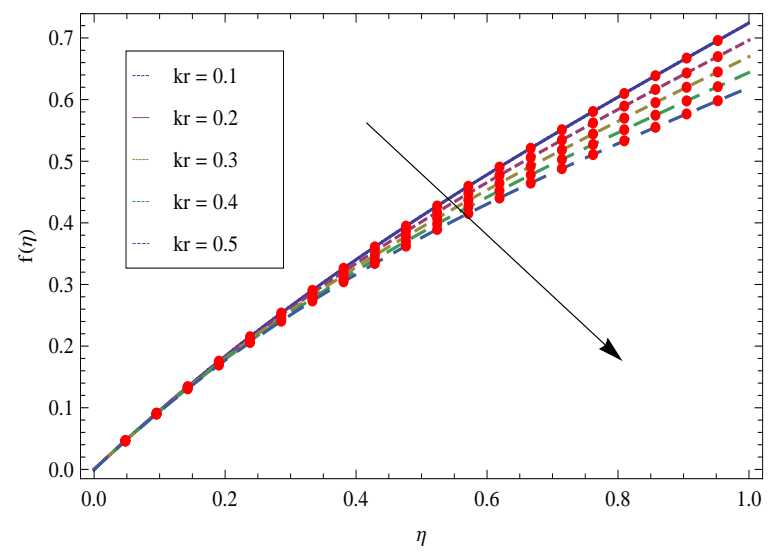

Figure 10. Indicates the effect of $K r$ on $f(\eta)$ for $h=-0.25, S=0.5, \operatorname{Pr}=0.5, \lambda=1$, Ec $=0.5$, $\mathrm{Nb}=0.5, \mathrm{Nt}=0.6, \beta=1, \mathrm{Sc}=0.6$.

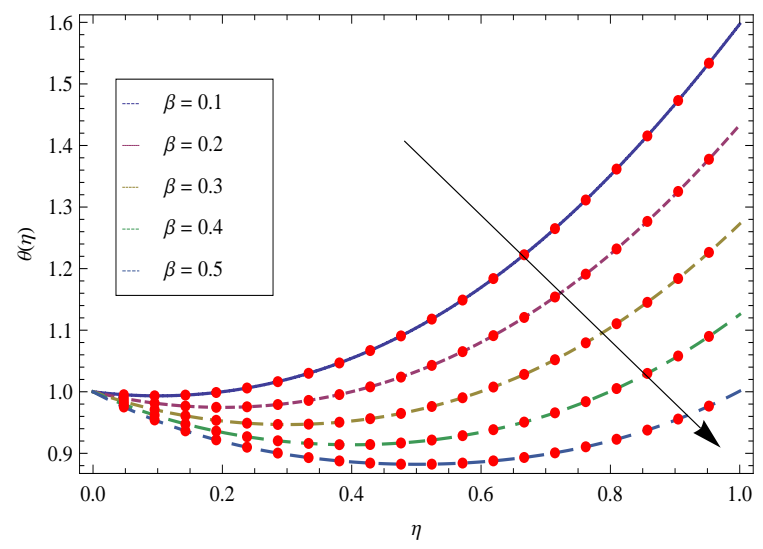

Figure 11. Shows the effect of $\beta$ on $\theta(\eta)$ for $h=-0.7, \lambda=1, S=0.5, \mathrm{kr}=0.5, \operatorname{Pr}=0.5$, Ec $=0.5$, $\mathrm{Nb}=0.5, \mathrm{Nt}=0.6$, and $S c=0.6$. 


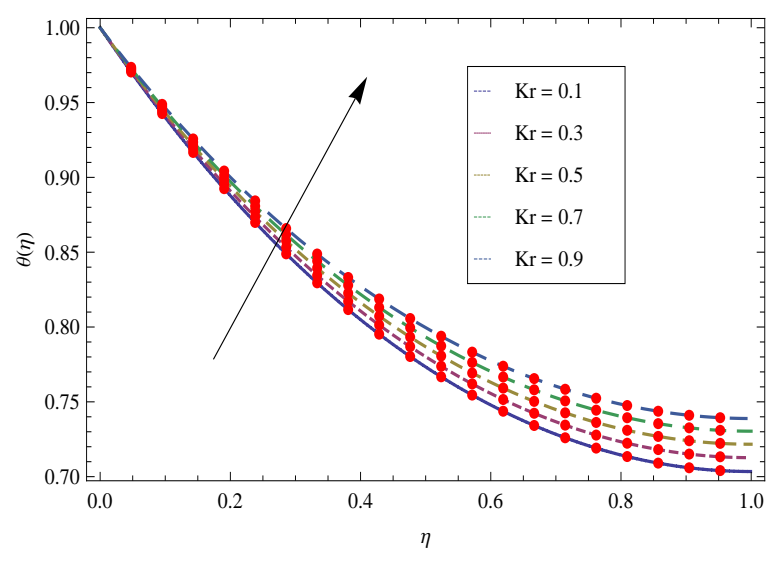

Figure 12. Shows the effect of $K r$ on $\theta(\eta)$ for $h=-0.25, \lambda=0.1, S=0.1, \operatorname{Pr}=0.5, \mathrm{Ec}=0.5, \mathrm{Nb}=0.5$, $\mathrm{Nt}=0.6$ and $S c=0.6$.

The effect of Prandtl number $\operatorname{Pr}$ has been shown in the Figure 13, describing that for larger values of $\operatorname{Pr}$ decreases the temperature $\theta(\eta)$. The increase in Prandtl number reduces the thermal boundary layer due to which the temperature decreases. The influence of the Schmidt number $S c$ is depicted in Figures 14 and 15, showing that temperature and concentration fields decrease when the parameter $S c$ increases because Schmidt number $S c$ is reciprocal to the molecular diffusivity. It indicates that as the values of of the Eckert number $E c$ increase the fluid temperature also increases while its converse effect has been observed in the solute concentration illustrated in Figures 16 and 17. Physically, Ec is connected with the viscous dissipation term in the equation of energy, therefore, larger values of $E c$ should lead to increase the quantity of heat being produced by the shear forces in the fluid and as a result raises the fluid temperature. Figures 18 and 19, illustrate the effects of Brownian motion parameter $\mathrm{Nb}$ on the dimensionless temperature and concentration profiles. The fluid temperature increases as the value of increase of Brownian motion parameter increase while converse effect on the solute concentration. The increase in the value of thermophoresis parameter, increase both temperature and concentration as illustrated in Figures 20 and 21. The fluid flow is also falling when the thickness of film is increased. Larger values of thickness $\beta$ generate the friction force and as a result the flow motion falls down. Increase in the film thickness deliver more fluid in the boundary layer region and cooling effect is produced, which absorbs the heat transfer from the sheet to the fluid and temperature profile drops down. Concentration has vital application in thermal conductivity and chemical reactions. The concentration profile $\phi(\xi)$ is reliant on film size $\beta$ and increases with larger values of $\beta$ indicated in Figures 22-24. The $h$-curves of $f^{\prime \prime}(0), \theta^{\prime}(0)$, and $\phi^{\prime}(0)$ for the 4th-order HAM approximated solution are elaborated in Figures 25-27. Figures 28-31 indicate $h$ curves of the residuals for velocity, temperature and concentration profiles respectively. Table 1 illustrates the symbols used in the manuscript. In Tables $2-4$ the results are compared, which are achieved by HAM and Numerical(ND-Solve method) for velocity, temperature and concentration profiles. The residuals gained by HAM are also depicted in Table 5 . 


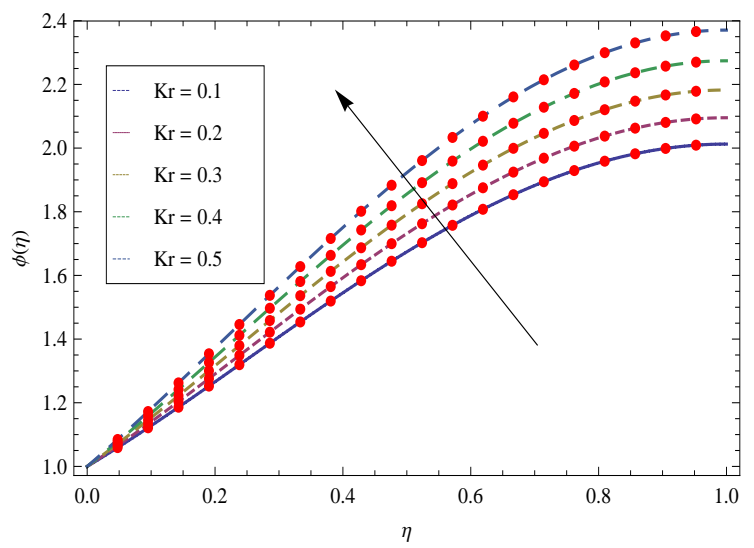

Figure 13. The effect of $K r$ on $\phi(\eta)$ for $h=-0.9, \lambda=0.5, S=0.7, \operatorname{Pr}=0.5, \mathrm{Ec}=0.5, \mathrm{Nb}=0.7$, $\mathrm{Nt}=0.1, \beta=1$ and $S c=0.5$.

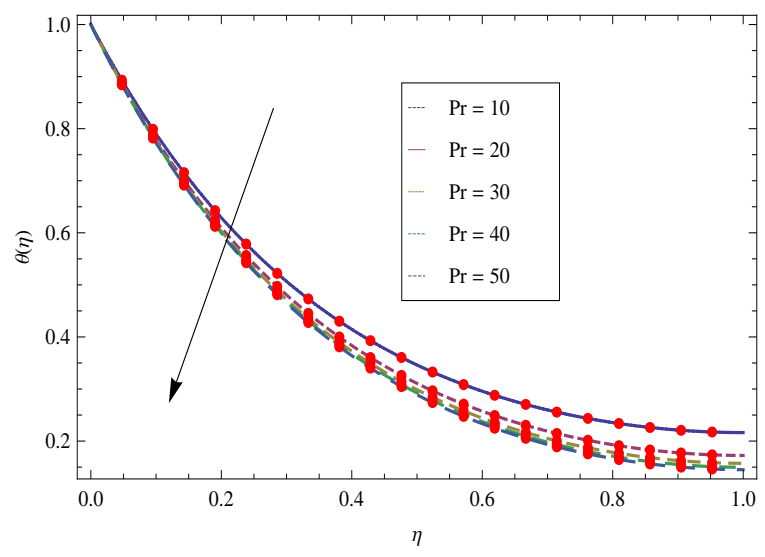

Figure 14. Shows the effect of $\operatorname{Pr}$ on $\theta(\eta)$ for $h=-0.7, \lambda=0.7, S=0.7, \mathrm{kr}=0.1, \mathrm{Ec}=0.5, \mathrm{Nb}=0.5$, $\mathrm{Nt}=0.2, \beta=1$ and $S c=0.2$.

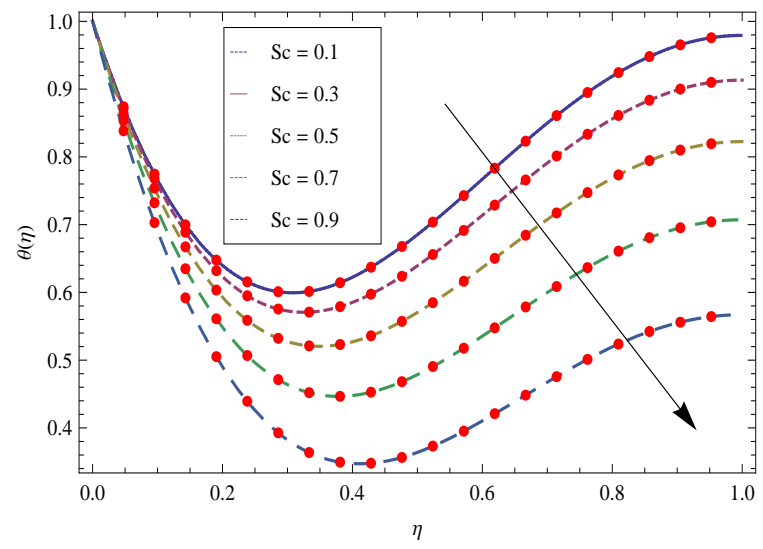

Figure 15. Shows the effect of $S c$ on $\theta(\eta)$ for $h=-0.9, \lambda=0.7, S=0.7, \mathrm{kr}=0.7, \operatorname{Pr}=30, \mathrm{Ec}=0.7$, $\mathrm{Nb}=0.5, \mathrm{Nt}=0.7, \beta=1$ and $S c=0.1$. 


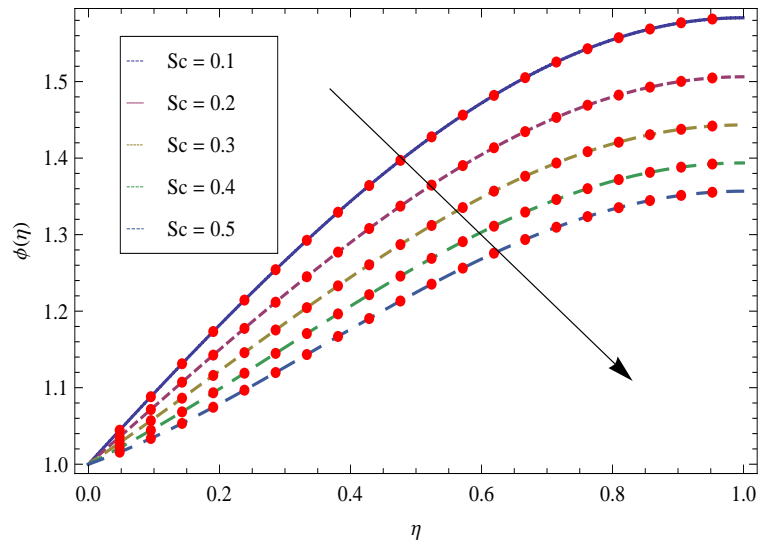

Figure 16. Shows the effect of $S c$ on $\phi(\eta)$, for $h=-0.6, \lambda=0.5, S=0.5, \mathrm{kr}=0.5, \operatorname{Pr}=0.5$, Ec $=0.5$, $\mathrm{Nb}=0.5, \mathrm{Nt}=0.5, \beta=1$ and $S c=0.6$.

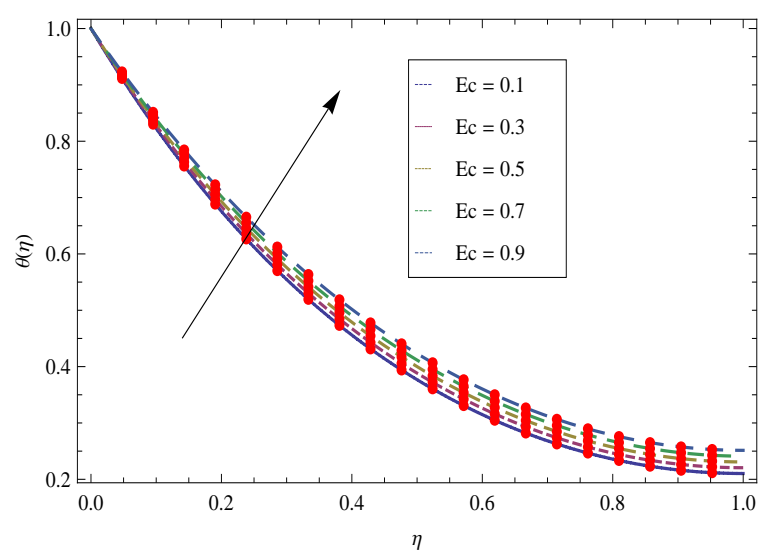

Figure 17. Shows the effect of $E_{c}$ on $\theta(\eta)$, for $h=-0.6, \lambda=0.1, S=0.1, \mathrm{kr}=0.9, \operatorname{Pr}=15, \mathrm{Nb}=0.5$, $\mathrm{Nt}=0.6, \beta=1$ and $S c=0.1$.

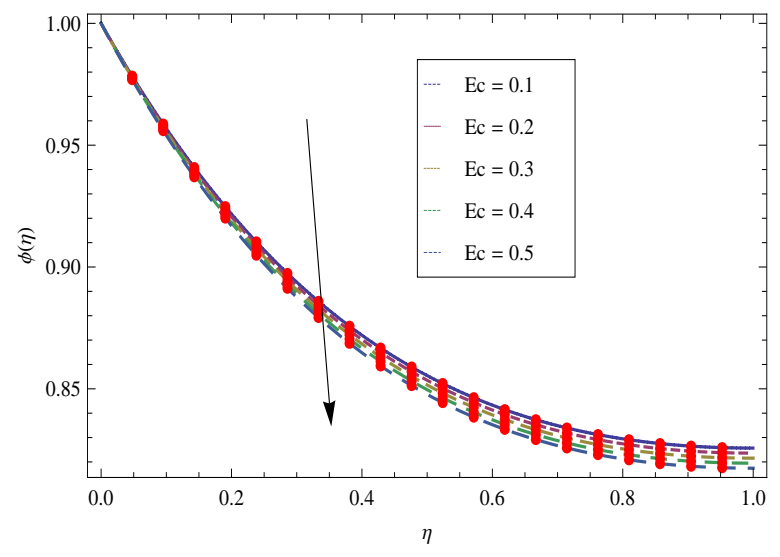

Figure 18. presents the effect of $E c$ on $\phi(\eta)$ for $h=-0.7, \lambda=0.5, S=0.7, \mathrm{kr}=0.2, \operatorname{Pr}=10, \mathrm{Nb}=0.7$, $\mathrm{Nt}=0.1, \beta=1$ and $S c=0.5$. 


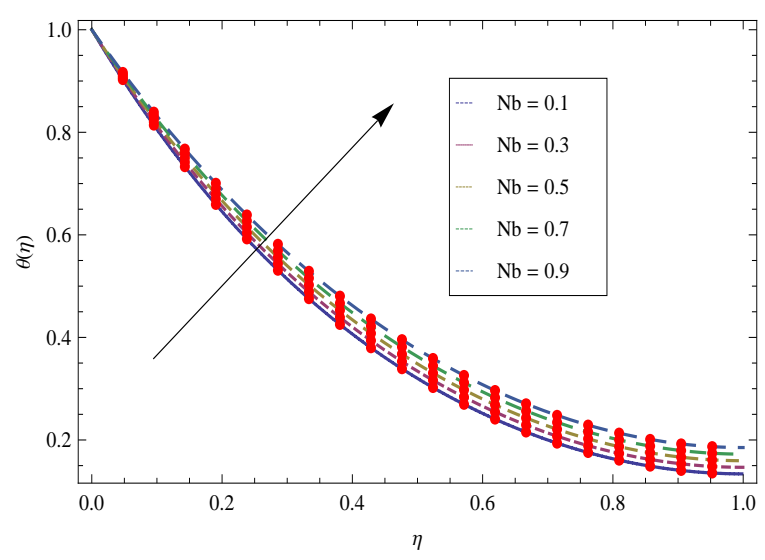

Figure 19. Illustrates the effect of $N b$ on $\theta(\eta)$, when $h=-0.5, \lambda=0.7, S=0.7, \mathrm{kr}=0.7, \operatorname{Pr}=30$, $\mathrm{Ec}=0.7, \mathrm{Nt}=0.5, \beta=1$ and $S c=0.7$.

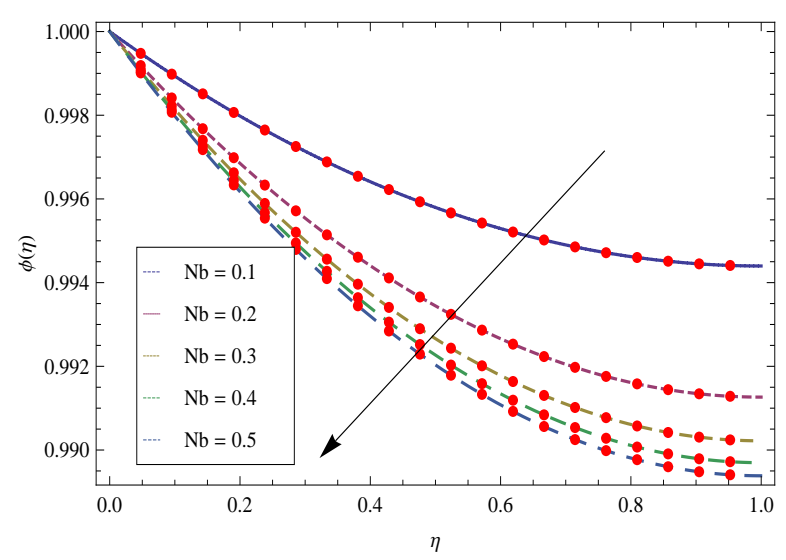

Figure 20. Indicates the effect of $N b$ on $\phi(\eta)$, for $h=-0.9, \lambda=0.5, S=0.7, \mathrm{kr}=0.5, \operatorname{Pr}=0.5$, $\mathrm{Ec}=0.5, \mathrm{Nt}=0.7, \beta=1$ and $S c=0.5$.

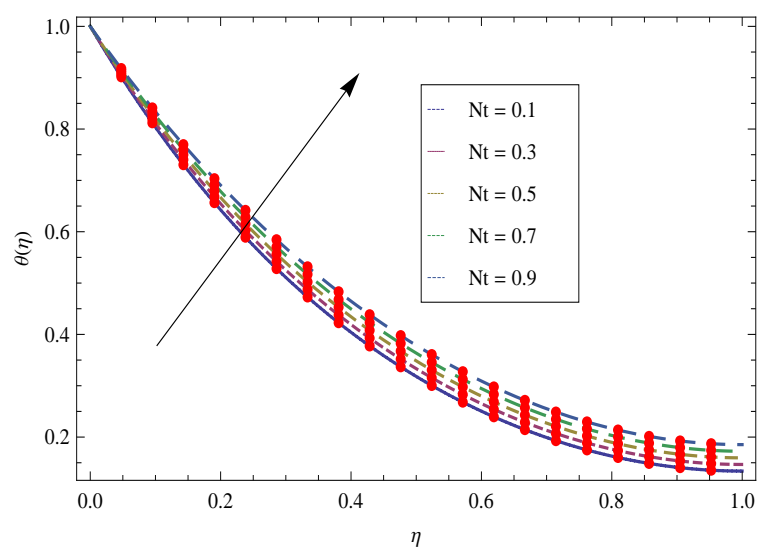

Figure 21. The effect of $N t$ on $\theta(\eta)$, when $h=-0.5, \lambda=0.7, S=0.7, \mathrm{kr}=0.7, \operatorname{Pr}=30, \mathrm{Ec}=0.7$, $\mathrm{Nb}=0.5, \beta=1$, and $S c=0.7$. 


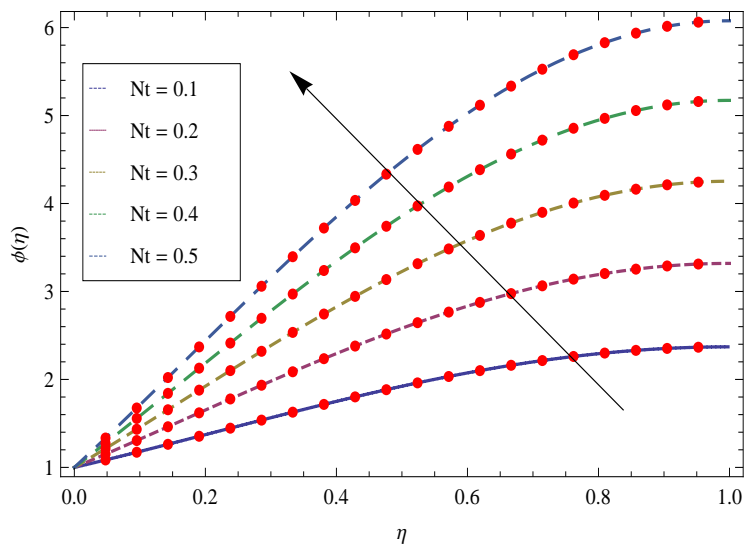

Figure 22. The effect of $N t$ on $\phi(\eta)$, for $h=-0.9, \lambda=0.5, S=0.7, \mathrm{kr}=0.5, \operatorname{Pr}=0.5, \mathrm{Ec}=0.5$, $\mathrm{Nb}=0.7, \beta=1$ and $S c=0.5$.

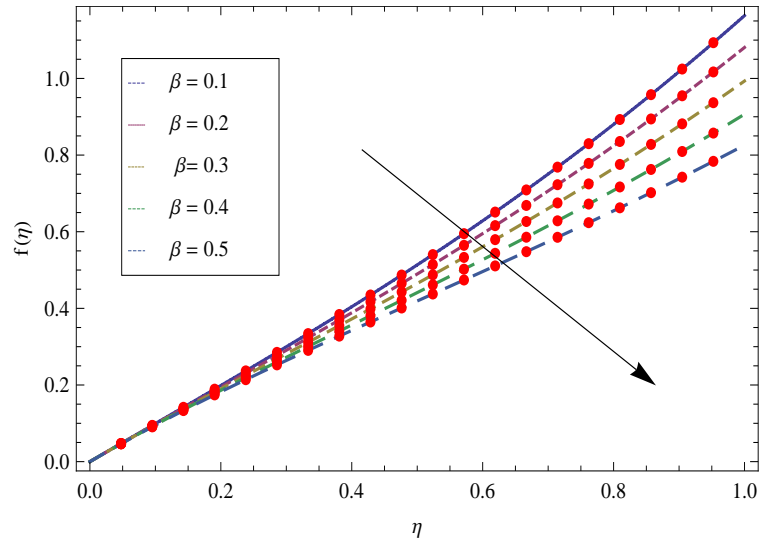

Figure 23. Shows the effect of $\beta$ on $f(\eta)$, for $h=-0.7, \lambda=1, \mathrm{kr}=0.5, \operatorname{Pr}=0.5, \mathrm{Ec}=0.5, \mathrm{Nb}=0.5$, $\mathrm{Nt}=0.6, \beta=0.1$ and $S c=0.6$.

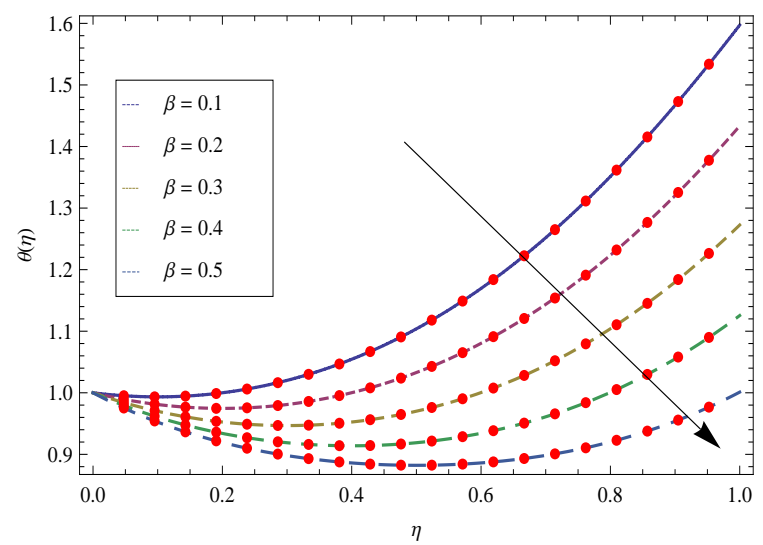

Figure 24. The effect of $\beta$ on $\theta(\eta)$, for $h=-0.7, \lambda=1, S=0.5, \mathrm{kr}=0.5, \mathrm{Ec}=0.5, \mathrm{Nb}=0.5, \mathrm{Nt}=0.6$ and $S c=0.6$. 


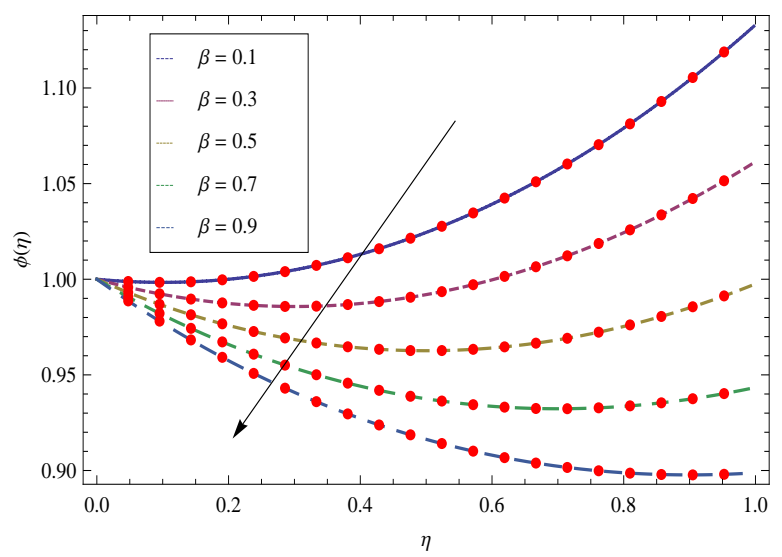

Figure 25. Illustrates the effect of $\beta$ on $\phi(\eta)$ for $h=-0.25, \lambda=0.5, S=0.1, \mathrm{kr}=0.5$, Ec $=0.5$, $\mathrm{Nb}=0.5, \mathrm{Nt}=0.6$ and $S c=0.6$.

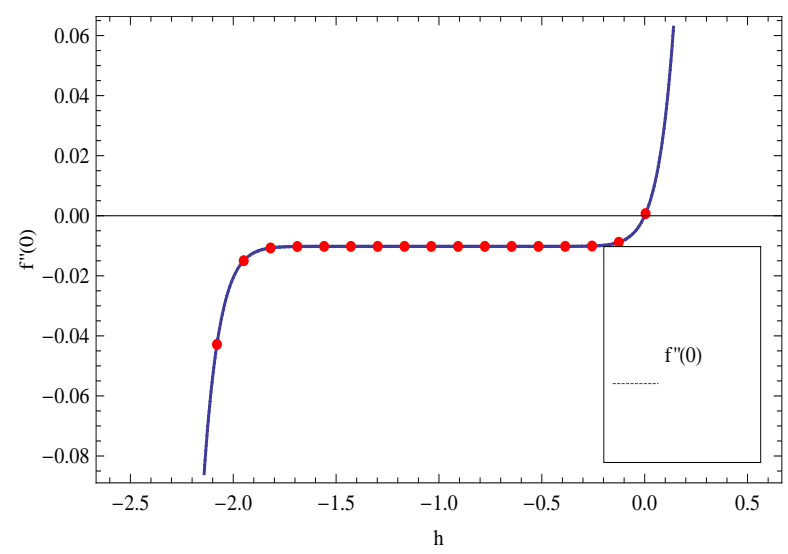

Figure 26. Depicts $h$ curves of $f^{\prime \prime}(0)$, when $\lambda=0.9, \mathrm{kr}=0.9, \mathrm{Pr}=0.5, \mathrm{Ec}=0.5, \mathrm{Nb}=0.5, \mathrm{Nt}=0.6$, $\beta=1$ and $S c=0.6$.

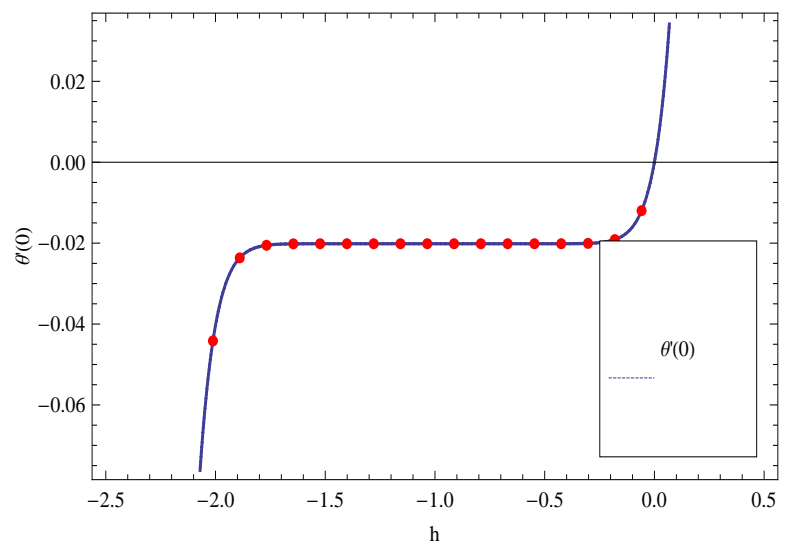

Figure 27. Shows $h$ curves of $\theta^{\prime}(0)$, for $\lambda=0.2, S=0.2, \mathrm{kr}=0.2, \operatorname{Pr}=1, \mathrm{Ec}=0.6, \mathrm{Nb}=1, \mathrm{Nt}=0.1$, $\beta=1$ and $S c=0.5$. 


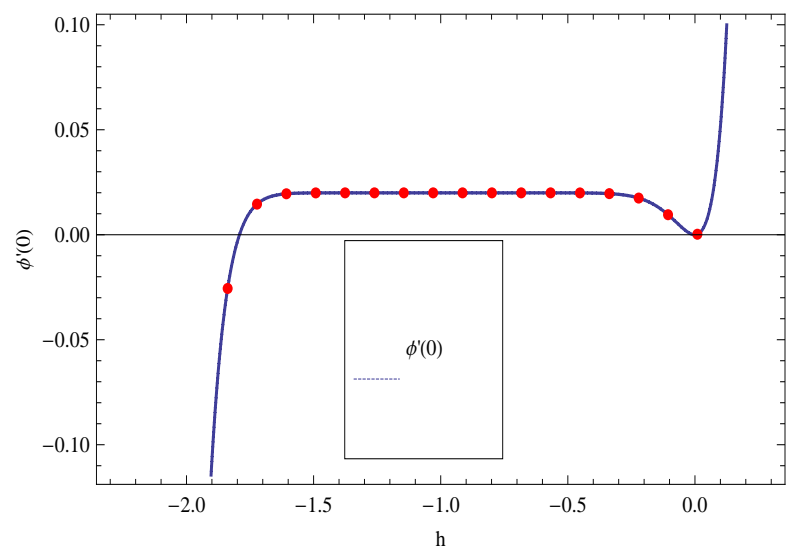

Figure 28. Elaborates $h$ curves of $\phi^{\prime}(0)$, when $\lambda=0.2, S=0.2, \mathrm{kr}=0.2, \operatorname{Pr}=1, \mathrm{Ec}=0.6, \mathrm{Nb}=0.4$, $\mathrm{Nt}=0.5, \beta=1, S c=0.5$.

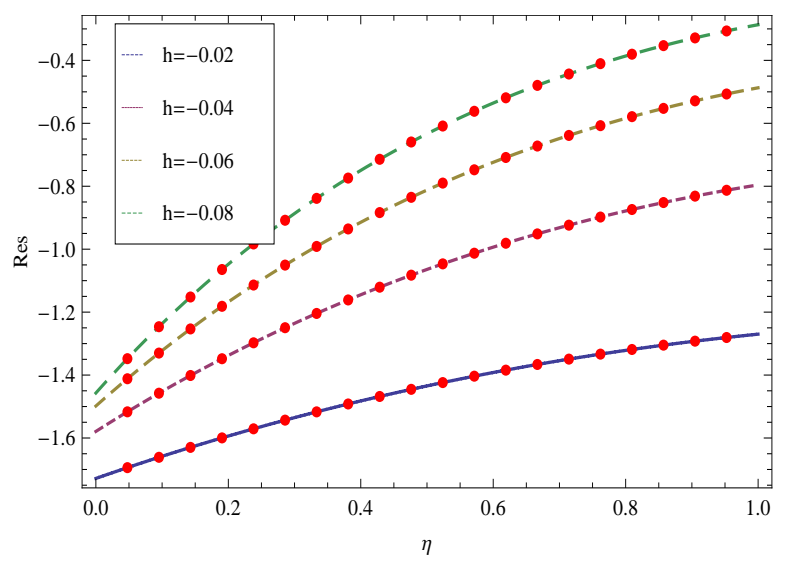

Figure 29. Illustrates $h$ curves of the residuals for the velocity profile $f(\eta)$, when $\lambda=0.6, \mathrm{~S}=0.6, \mathrm{Kr}=$ $0.4, \operatorname{Pr}=1, \mathrm{Ec}=0.4, \mathrm{Nb}=0.6, \mathrm{Nt}=0.5, \mathrm{Sc}=0.5, \beta=1$.

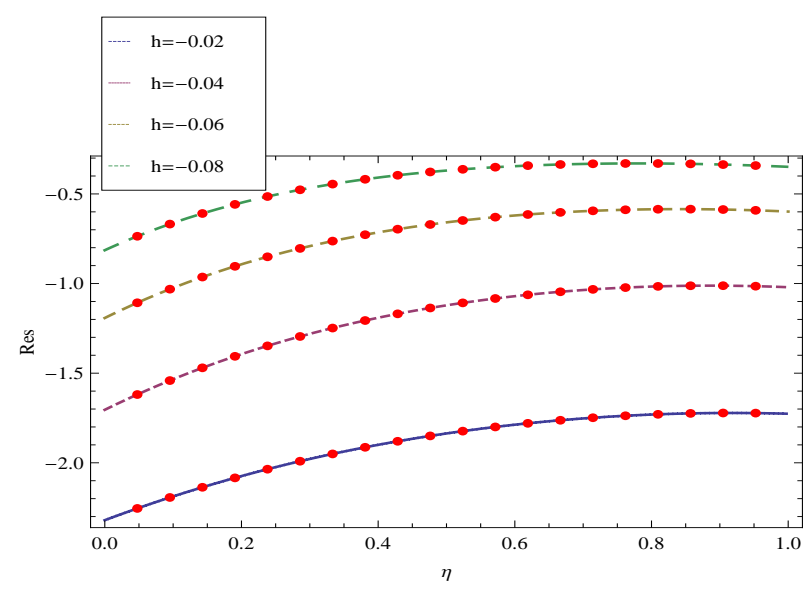

Figure 30. Indicates $h$ curves of the residuals for the temperature profile $\theta(\eta)$, when $\lambda=0.6, S=0.6$, $\mathrm{Kr}=0.4, \mathrm{Pr}=1, \mathrm{Ec}=0.4, \mathrm{Nb}=0.6, \mathrm{Nt}=0.5, \mathrm{Sc}=0.5, \beta=1$. 


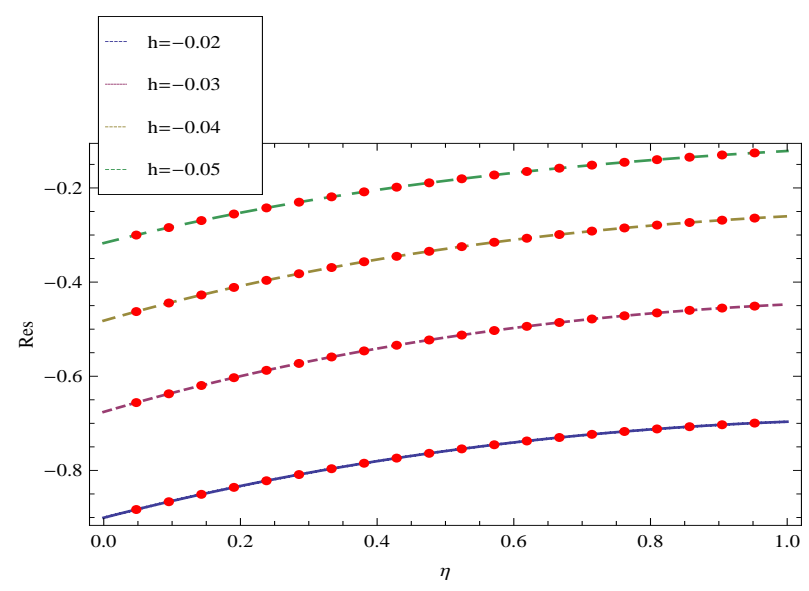

Figure 31. Shows $h$ curves of the residuals for the concentration profile $\phi(\eta)$, when $\lambda=0.6, s=0.6$; $\mathrm{Kr}=0.4, \mathrm{Pr}=1, \mathrm{Ec}=0.4, \mathrm{Nb}=0.6, \mathrm{Nt}=0.5, \mathrm{sc}=0.5, \beta=1$.

Table 1. Shows the Nomenclature.

\begin{tabular}{|c|c|c|c|}
\hline Alphabet & Defined as & Alphabet & Defined as \\
\hline$y$ & vertical coordinate $(m)$ & $T_{0}$ & initial temperature of the fluid $(\mathrm{K})$ \\
\hline$v$ & vertical velocity component $(\mathrm{m} / \mathrm{s})$ & $U_{w}$ & Velocity of the stretching sheet \\
\hline$S$ & Unsteadiness parameter & $T_{1}$ & final temperature of the fluid $(\mathrm{K})$ \\
\hline$T_{w}$ & Temperature at the sheet & $\mathrm{T}$ & temperature $(\mathrm{K})$ \\
\hline$b$ & stretching parameter(constant) & $\mathrm{k}^{\prime}$ & permeability coefficient of the porosity \\
\hline$f(\xi)$ & nondimensional variable for velocity & $C_{w}$ & Nanoparticle volume fraction at sheet \\
\hline $\operatorname{Pr}$ & Prandtl number & $E c$ & Eckert number \\
\hline$K r$ & nondimensional porosity parameter & Sc & Schmidt number \\
\hline $\mathrm{Nb}$ & Brownian motion parameter & $D_{B}$ & Brownian diffusion coefficient \\
\hline$C_{p}$ & specific heat at constant pressure $\left(\mathrm{kJ} \mathrm{kg}^{-1} \mathrm{~K}^{-1}\right)$ & $N t$ & Thermophoresis parameter \\
\hline$\rho$ & density $\left(\mathrm{kg} \mathrm{m}^{-3}\right)$ & $(\rho c)_{p}$ & Heat capacity of the nanoparticle material \\
\hline$\Gamma$ & Time constant & $\alpha$ & Thermal diffusivity of the base fluid \\
\hline$\rho_{p}$ & Nanoparticle mass density & $(\rho c)_{f}$ & Heat capacity of the base fluid \\
\hline$v$ & Kinematic viscosity of the base fluid & $\lambda$ & Williamson fluid constant \\
\hline$\beta$ & non-dimensional film thickness & $\theta$ & Dimensionless temperature \\
\hline$\psi$ & non-dimensional stream function & $\left({ }^{\prime}\right)$ & differentiation w. r. t. $\xi$ \\
\hline
\end{tabular}

Table 2. HAM, Numerical Solution and their absolute Error are shown for $f(\eta)$, when $h=-0.47$, $\lambda=0.2, \mathrm{kr}=0.2, \operatorname{Pr}=1, \mathrm{Ec}=0.6, \mathrm{Nb}=0.4, \mathrm{Nt}=0.5, S=0.2, \beta=1$ and $S c=0.5$.

\begin{tabular}{cccc}
\hline $\boldsymbol{\eta}$ & $\begin{array}{c}\text { Numerical Solution for } \\
\boldsymbol{f}(\boldsymbol{\eta})\end{array}$ & $\begin{array}{c}\text { HAM Solution for } \\
\boldsymbol{f}(\boldsymbol{\eta})\end{array}$ & Absolute Error \\
\hline 0 & 0 & 0 & 0 \\
0.1 & 0.0952761 & 0.0955184 & $2.4 \times 10^{-4}$ \\
0.2 & 0.182152 & 0.182958 & $8.1 \times 10^{-4}$ \\
0.3 & 0.262045 & 0.263561 & $1.5 \times 10^{-3}$ \\
0.4 & 0.336198 & 0.338462 & $2.3 \times 10^{-3}$ \\
0.5 & 0.405709 & 0.408698 & $2.9 \times 10^{-3}$ \\
0.6 & 0.471556 & 0.47522 & $3.6 \times 10^{-3}$ \\
0.7 & 0.534618 & 0.5389 & $4.3 \times 10^{-3}$ \\
0.8 & 0.595686 & 0.600535 & $4.6 \times 10^{-3}$ \\
0.9 & 0.655482 & 0.660862 & $5.4 \times 10^{-3}$ \\
1 & 0.714666 & 0.720558 & $5.8 \times 10^{-3}$ \\
\hline
\end{tabular}


Table 3. HAM, Numerical Solution and their absolute Error are elobarated for $\theta(\eta)$, when $h=-0.5$, $\lambda=0.2, \mathrm{kr}=0.2, \operatorname{Pr}=0.5, \mathrm{Ec}=0.6, \mathrm{Nb}=0.4, \mathrm{Nt}=0.5, S=0.2, \beta=1$ and $S c=0.7$.

\begin{tabular}{cccc}
\hline $\boldsymbol{\eta}$ & $\begin{array}{c}\text { Numerical Solution for } \\
\boldsymbol{\theta}(\boldsymbol{\eta})\end{array}$ & $\begin{array}{c}\text { HAM Solution for } \\
\boldsymbol{\theta}(\boldsymbol{\eta})\end{array}$ & Absolute Error \\
\hline 0 & 1 & 1 & $1.4 \times 10^{-8}$ \\
0.1 & 0.907342 & 0.90737 & $2.8 \times 10^{-5}$ \\
0.2 & 0.82775 & 0.827596 & $1.5 \times 10^{-4}$ \\
0.3 & 0.759861 & 0.759482 & $3.8 \times 10^{-4}$ \\
0.4 & 0.702621 & 0.702067 & $5.5 \times 10^{-4}$ \\
0.5 & 0.655233 & 0.654586 & $6.5 \times 10^{-4}$ \\
0.6 & 0.617098 & 0.616438 & $6.7 \times 10^{-4}$ \\
0.7 & 0.587786 & 0.587169 & $6.2 \times 10^{-4}$ \\
0.8 & 0.567002 & 0.566451 & $5.5 \times 10^{-4}$ \\
0.9 & 0.554573 & 0.554078 & $4.9 \times 10^{-4}$ \\
1 & 0.550428 & 0.549956 & $4.7 \times 10^{-4}$ \\
\hline
\end{tabular}

Table 4. HAM, Numerical Solution and their absolute Error are depicted for $\phi(\eta)$, when $h=-0.6$, $\lambda=0.2, \mathrm{kr}=0.2, \operatorname{Pr}=1, \mathrm{Ec}=0.6, \mathrm{Nb}=1, \mathrm{Nt}=0.1, S=0.2, \beta=1$, and $S c=0.5$.

\begin{tabular}{cccc}
\hline $\boldsymbol{\eta}$ & $\begin{array}{c}\text { Numerical Solution for } \\
\boldsymbol{\phi}(\boldsymbol{\eta})\end{array}$ & $\begin{array}{c}\text { HAM Solution for } \\
\boldsymbol{\phi}(\boldsymbol{\eta})\end{array}$ & Absolute Error \\
\hline 0 & 1 & 1 & $2.9 \times 10^{-9}$ \\
0.1 & 0.940581 & 0.941167 & $5.9 \times 10^{-4}$ \\
0.2 & 0.890316 & 0.8912 & $8.8 \times 10^{-4}$ \\
0.3 & 0.848224 & 0.849209 & $9.8 \times 10^{-4}$ \\
0.4 & 0.813465 & 0.81443 & $9.6 \times 10^{-4}$ \\
0.5 & 0.785325 & 0.786207 & $8.8 \times 10^{-4}$ \\
0.6 & 0.763201 & 0.76398 & $7.8 \times 10^{-4}$ \\
0.7 & 0.74659 & 0.747277 & $6.9 \times 10^{-4}$ \\
0.8 & 0.735082 & 0.735705 & $6.2 \times 10^{-4}$ \\
0.9 & 0.728352 & 0.728942 & $5.9 \times 10^{-4}$ \\
1 & 0.726151 & 0.726734 & $5.8 \times 10^{-4}$ \\
\hline
\end{tabular}

Table 5. Illustrates the residuals achieved by HAM for system of coupled differential equations forming in velocity, temperature and concentration profiles.

\begin{tabular}{cccc}
\hline $\boldsymbol{\eta}$ & $\begin{array}{c}\text { Residuals for } \\
\boldsymbol{f}(\boldsymbol{\eta})\end{array}$ & $\begin{array}{c}\text { Residuals for } \\
\boldsymbol{\theta}(\boldsymbol{\eta})\end{array}$ & $\begin{array}{c}\text { Residuals for } \\
\boldsymbol{\phi}(\boldsymbol{\eta})\end{array}$ \\
\hline 0 & $-2.0 \times 10^{-1}$ & $-3.7 \times 10^{-1}$ & $5.1 \times 10^{-2}$ \\
0.1 & $-1.2 \times 10^{-1}$ & $-7.9 \times 10^{-2}$ & $2.4 \times 10^{-2}$ \\
0.2 & $-4.9 \times 10^{-2}$ & $3.2 \times 10^{-3}$ & $2.2 \times 10^{-2}$ \\
0.3 & $-4.9 \times 10^{-4}$ & $2.5 \times 10^{-2}$ & $2.9 \times 10^{-2}$ \\
0.4 & $3.3 \times 10^{-2}$ & $2.9 \times 10^{-2}$ & $3.2 \times 10^{-2}$ \\
0.5 & $5.2 \times 10^{-2}$ & $2.1 \times 10^{-2}$ & $2.3 \times 10^{-2}$ \\
0.6 & $6.1 \times 10^{-2}$ & $5.9 \times 10^{-3}$ & $2.2 \times 10^{-3}$ \\
0.7 & $5.9 \times 10^{-2}$ & $-1.3 \times 10^{-2}$ & $-2.6 \times 10^{-2}$ \\
0.8 & $4.9 \times 10^{-2}$ & $-3.3 \times 10^{-2}$ & $-5.5 \times 10^{-2}$ \\
0.9 & $3.3 \times 10^{-2}$ & $-4.6 \times 10^{-2}$ & $-7.3 \times 10^{-2}$ \\
1 & $9.9 \times 10^{-3}$ & $-4.9 \times 10^{-2}$ & $-8.7 \times 10^{-2}$ \\
\hline
\end{tabular}

\section{Conclusions}

The main conclusion of this endeavor is the study of liquid film in a porous medium considering non-Newtonian Williamson fluid on an unstable stretching surface. The effect of Thermophoresis and Brownian motion has been countered to the liquid film flow. The solutions of the problems have 
been achieved by using analytical technique, HAM for velocity, temperature and concentration fields respectively. The influences of all parameters included in the problem have been described and the solutions are displayed in the diagrams for checking their effects on velocity, temperature as well as concentration fields. The coupled problem has been solved by using an analytical method HAM. The $h$ curves for the residuals of velocity, temperature and concentration have been sketched.

The main concluded points are derived as,

(1) Increasing thickness parameter $\beta$ produce the friction force and as a result velocity of the fluid film falls down.

(2) The larger values of $\beta$ transport more fluid in the boundary layer region and cooling effect is produced which absorbed the heat transfer from the sheet and as a result the temperature reduces.

(3) The Eckert number $E c$ is allied with the viscous dissipation term and lead to incrrease the quantity of heat being produced by the shear forces in the fluid. Therefore, larger values of $E c$ raises the temperature field.

(4) The larger values of Prandtl number $\mathrm{Pr}$ reduces the thermal boundary layer due to which the temperature field reduces.

(5) Higher values of Porosity parameter $K r$ generate larger open space and create hurdle to flow and as a result the flow field reduces.

Author Contributions: Liaqat Ali, Taza Gul and Waris Khan modeled the problem and solved it; Saeed Islam, Liaqat Ali, and Taza Gul contributed to the discussion of the problem; L.C.C.Dennis, Saeed Islam, Ilyas Khan and Aurangzeb Khan contributed in the English corrections. All the authors read and approved the final manuscript.

Conflicts of Interest: The authors declare no conflict of interest.

\section{References}

1. Williamson, R.W. The dyanamics of lava flows. Annu. Rev. Fluid Mech. 2000, 32, 477-518.

2. Dapra, I.; Scarpi, G. Perturbation solution for pulsatile flow of a non newtonian Williamson fluids in rock fracture. Int. J. Rock Mech. Min. Sci. 2006, 44, 1-8.

3. Wang, C.Y. Liquid film on an unsteady stretching surface. Q. Appl. Math. 1990, 48, 601-610.

4. Cramer, K.; Pai, S. Magneto Fluid Dynamics for Engineers and Applied Physicists; McGraw-Hill: New York, NY, USA, 1973.

5. Selim, A.; Hossain, M.A.; Rees, D.A.S. The effect of surface mass transfer mixed convection flow past a heated vertical flat permeable plate with thermophoresis. Int. J. Therm. Sci 2003, 42, 973-982.

6. Das, K. Impact of thermal radiation on MHD slip flow over a flat plate with variable fluid properties. Heat Mass. Transf. 2012, 48, 767-778.

7. Siddeshwar, P.G; Mahabaleshwar, U.S. Effects of radiation and heat transfer on MHD flow of viscoelastic liquid and heat transfer over a stretching sheet. Int. J. Nonlinear Mech. 2005, 40, 807-820.

8. Nadeem, S.; Hussain, S.T. Flow and heat transfer analysis of Williamson nanofluid. Appl. Nanosci. 2014, 4, 1005-1012.

9. Hassanein, I.A.; Essawy, A.; Morsy, N.M. Variable viscosity and thermal conductivity effects on heat transfer by natural convection from a cone and a wedge in porous media. Arch. Mech. 2004, 55, 345-356.

10. Aziz, R.C.; Hashim, I.; Alomari, A.K. Thin film flow and heat transfer on an unsteady stretching sheet with internal heating. Meccanica 2011, 46, 349-357.

11. Qasim, M.; Khan, Z.H.; Lopez, R.J.; Khan, W.A. Heat and mass transfer in nanofluid over an unsteady stretching sheet using Buongiorno's model. Eur. Phys. J. Plus 2016, 131, 1-16.

12. Mahesh, K.; Gireesha, B.J;; Rama, S.R.G. Heat and Mass Transfer in Nanofuid over an unsteady stretching surface. J. Nanofluids 2015, 4, 1-8.

13. Ellahi, R.; Hassan, M.; Zeeshan, A. Aggregation effects on water base $\mathrm{Al}_{2} \mathrm{O}_{3}$-Nanofluid over permeable wedge in mixed convection. Asia-Pac. J. Chem. Eng. 2016, 11, 179-186.

14. Akbar, N.S.; Raza, M.; Ellahi, $\mathrm{R}$. CNT suspended $\mathrm{CuO}+\mathrm{H}_{2} \mathrm{O}$ nano fluid and energy analysis for the peristaltic flow in a permeable channel. Eng. J. 2015, 54, 623-633.

15. Akbar, N.S.; Raza, M.; Ellahi, R. Copper oxide nanoparticles analysis with water as base fluid for peristaltic flow in permeable tube with heat transfer. Comput. Methods Progr. Biomed. 2016, 130, 22-30. 
16. Shehzad, N.; Zeeshan, A.; Ellahi, R.; Vafai, K. Convective heat transfer of nanofluid in a wavy channel: Buongiorno's mathematical model. J. Mol. Liq. 2016, 222, 446-455.

17. Zeeshan, A.; Hassan, M.; Ellahi, R.; Nawaz, M. Shape effect of nanosize particles in unsteady mixed convection flow of nanofluid over disk with entropy generation. J. Process Mech. Eng. 2016, doi:10.1177/0954408916646139.

18. Khan, W.; Gul, T.; Idrees, M.; Islam, I.; Khan, I.; Dennis, L.C.C. Thin Film Williamson Nanofluid Flow with Varying Viscosity and Thermal Conductivity on a Time-Dependent Stretching Sheet. Appl. Sci. 2016, 6, 334, doi:10.3390/app6110334.

19. Gamal, M.; Rahman, A. Effect of Magnetohydrodynamic on Thin Films of Unsteady Micropolar Fluid through a Porous Medium. J. Mod. Phys. 2011, 2, 1290-1304 .

20. Jaina, S.; Choudhary, R. Effects of MHD on Boundary Layer Flow in Porous Medium due to Exponentially Shrinking Sheet with Slip. Procedia Eng. 2015 , 127, 1203-1210.

21. Liao, S. Beyond Perturbation: Introduction to the Homotopy Analysis Method; Chapman and Hall/CRC: Boca Raton, FL, USA, 2003.

22. Liao, S.J. An optimal homotopy-analysis approach for strongly nonlinear differential equations. Commun. Nonlinear Sci. Numer. Simul. 2010, 15, 2003-2016.

23. Liao, S. On the homotopy analysis method for nonlinear problems. Appl. Math. Comput. 2004, 147, 499-513.

24. Abbasbandy, S.; Shirzadi, A. A new application of the homotopy analysis method: Solving the Sturm-Liouville problems. Commun. Nonlinear Sci. Numer. Simul. 2011, 16, 112-126.

25. Abbasbandy, S. Homotopy analysis method for heat radiation equations. Int. Commun. Heat Mass Transf. 2007, 34, 380-388.

26. Abbasbandy, $\mathrm{S}$. The application of homotopy analysis method to solve a generalized Hirota-Satsuma coupled KdV equation. Phys. Lett. A 2007, 361, 478-483.

27. Khan, N.S.; Taza Gul, T.; Islam, S.; Khan, W. Thermophoresis and thermal radiation with heat and mass transfer in a magnetohydrodynamic thin film second grade fluid of variable properties past a stretching sheet. Eur. Phys. J. Plus 2017, 132, doi:10.1140/epjp/i2017-11277-3.

28. Khan, W.; Gul, T.; Idrees, M.; Khan, I. Dufour and Soret Effect with Thermal Radiation on the Nano Film Flow of Williamson Fluid Past Over an Unsteady Stretching Sheet. J. Nanofluids 2017, 6, 243-253.

29. Narayana, M.; Sibanda, P. Hydromagnetic nanofluid flow due to a stretching or shrinking sheet with viscous dissipation and chemical reaction effects. Int. J. Heat Mass. Transf. 2012, 55, 7587-7595.

30. Xu, H.; Pop, I.; You, X.C. Flow and heat transfer in a nano-liquid film over an unsteady stretch surface. Int. J. Heat Mass. Transf. 2013, 60, 646-652.

31. Nadeem, S.; Haq, R.U.; Lee, C. MHD flow of a Casson fluid over an exponentially shrinking sheet. Sci. Iran. 2012, 19, 1550-1553.

32. Nadeem, S.; Haq, R.U.; Akbar, N.S.; Khan, Z.H. MHD three-dimensional Casson fluid flow past a porous linearly stretching sheet. Alex. Eng. J. 2013, 52, 577-582.

33. Rehman, S.U.; Haq, R.U.; Lee, C.; Nadeem, S. Numerical study of non-Newtonian fluid flow over an exponentially stretching surface: an optimal HAM validation. Braz. Soc. Mech. Sci. Eng. 2016, 7, doi:10.1007/s40430-016-0687-3.

34. Haq, R.U.; Shahzad, F.; Al-Mdallal, Q.M. MHD Pulsatile Flow of Engine oil based Carbon Nanotubes between Two Concentric Cylinders. Results Phys. 2016, 7, 57-68.

35. Besthapu, P.; Haq, R.U.; Bandari, S.; Al-Mdallal, Q.M. Mixed convection flow of thermally stratified MHD nanofluid over an exponentially stretching surface with viscous dissipation effect. J. Taiwan Inst. Chem. Eng. 2017, 71, 307-314.

(C) 2017 by the authors. Licensee MDPI, Basel, Switzerland. This article is an open access article distributed under the terms and conditions of the Creative Commons Attribution (CC BY) license (http:/ / creativecommons.org/licenses/by/4.0/). 